DOI:10.2478/rrlm-2019-0010

\title{
Do we really need genetic tests in current clinical practice?
}

\author{
Claudia Bănescu*
}

\author{
Laboratory of Molecular Biology/Genetics Center for Advanced Medical and Pharmaceutical \\ Research, University of Medicine, Pharmacy, Sciences and Technology of Târgu Mureş, Romania
}

Received: 27th December 2018; Accepted: $7^{\text {th }}$ January 2019; Published: $9^{\text {th }}$ January 2019

Currently there is an increased number of genetic tests available for disorders produced by chromosomal or gene abnormalities. Some genetic tests are recommended by healthcare providers, but some of them are represented by direct-to-consumer genetic testing, therefore knowing the applications and limitations of these tests is useful.

\section{Cytogenetic investigation in the diagnosis of chromosomal syndromes and leukemia}

Cytogenetic analysis used to be considered the gold-standard diagnostic tool to study chromosomal abnormalities (numerical and structural) in chromosomal disorders and in hematologic malignancies for a long time. Cytogenetic followed by karyotyping gives us a view of the entire genome, but at a low resolution $(\leq 5 \mathrm{Mb})$ and we may visualize individual cells and individual chromosomes.

Cytogenetic analysis of peripheral blood at 72 hours of cultures should be performed in cases suspected of chromosomal disorder, while cytogenetic analysis of bone marrow at 24 and
48 hours of short-term cultures should be performed in leukemia. For evaluation of chromosome number and morphology, GTG banding (G-banding by using trypsin for digestion and Giemsa staining) should be applied. At least 20 metaphases should be counted and karyotypes should be reviewed in compliance with the latest International System for Cytogenetic Nomenclature (ISCN) recommendations (1).

Some rather pessimistic people considered cytogenetics to be outdated, but the reality is different. The use of cytogenetic analysis especially in hematologic malignancies has allowed identification of an increased number of recurrent numerical and structural chromosomal abnormalities. The 2016 World Health Organization (WHO) classification of myeloid neoplasms and acute leukemia reinforced the value of genetic testing, including cytogenetic (2). In addition, the 2017 European Leukemia Net (ELN) recommendations for diagnosis and management of acute myeloid leukemia (AML) emphasized the importance of genetic investigation results (cytogenetic and molecular technologies) for risk

\footnotetext{
*Corresponding author: Claudia Bănescu, The University of Medicine, Pharmacy, Science, and Technology of Târgu Mureș, Romania. E-mail: claudia.banescu@rrml.ro
} 
stratification of patients with AML by genetics (3). Moreover, Döhner et al. considered that cytogenetic investigation remains mandatory in the evaluation of patients suspected of having AML. Unfortunately, cytogenetic analysis may be performed on mitotic cells and it is time-consuming.

\section{Fluorescent in situ hybridization (FISH)}

FISH is a molecular cytogenetic method that may be performed on interphase cells, it has a higher resolution that cytogenetic test $(\sim 100 \mathrm{~kb}$ to $1 \mathrm{Mb}$ ) and allows detection of chromosomal aberrations that cannot be identified by cytogenetic analysis (for example microdeletion or microduplication, balanced translocations) or when mitotic cells are not available for chromosomal analysis. Also, the FISH technique may be used to detect aneuploidies and balanced translocations associated with fusion genes, which are recurrent in acute and chronic myeloid leukemia (AML and CML). This method involves the hybridization of a given fluorescent probe (fluorescently labelled specific DNA sequence probe) with patient sample (DNA), followed by microscopic analysis for investigation of the presence, loss, gain of a chromosomal region).

There is an increased number of different types of fluorescent probes (FISH probes) that may be applied for molecular characterization: painting probes, locus specific probes (for example $\mathrm{BCR} / \mathrm{ABL}$ dual color, dual fusion translocation probes; break apart probes; subtelomeric probes), centromeric probes. FISH analysis allows a better description of changes identified by banding cytogenetics, as well as the discovery of new cryptic aberrations (deletion, duplication, fusion gene) (4).

The FISH method is also used for confirmation of the origin/ provenance of small supernumerary marker chromosome (sSMC) identified by cytogenetic testing (5).

\section{Array comparative genomic hybridization: array-CGH and SNP array}

Array CGH compares the patient's genome against a normal control genome (reference) and enables identification of structural aberrations such as copy number variations (CNVs, CNV is segment of DNA bigger than 1000 bases or $1 \mathrm{~Kb}$ which is present in a variable number of copies in comparison with standard DNA) or chromosomal imbalances. Array-CGH allows the screening of the entire genome, at a high resolution, thus being a useful tool in cases unexplained by karyotyping/ FISH, and also in AML patients with a complex karyotype and plays an important role in the accurate description of chromosomal anomalies (4).

Array-CGH has some limitations because it cannot detect balanced chromosomal rearrangements (such as balanced translocations, reciprocal insertions, inversions), point mutations, also it cannot identify chromosomal copy number changes, either gains or losses in regions of the genome that are not covered by the array, and cannot give data about parental origins of aneuploidies.

SNP array (Single nucleotide polymorphism array) is used to identify the presence of SNPs (a SNP being described as a variation in one nucleotide that occurs at a specific position in DNA sequence) among individuals. SNP array technique is similar to that of the array $\mathrm{CGH}$ but it is based on the investigation of thousands of SNPs. SNP array may be useful for identification of both CNVs and LOH (loss of heterozygosity), but also for detection of copy-neutral LOH (also known as uniparental disomy) and it should be considered in cases with syndromic or non-syndromic disorders that may be caused by a genomic copy number imbalance, and may also 
allow to identify the parental origin of aneuploidies. SNP array allows identification of several recurrent genetic abnormalities and gene mutations in hematologic malignancies.

\section{PCR (Polymerase chain reaction)-based techniques}

PCR is one of the most used and well-known methods in molecular biology for research but also for disease diagnosing. There are common PCR methods that can be used such as: nested PCR, PCR-RFLP, ARMS-PCR, RT-PCR, real-time PCR, etc.

\section{Polymerase chain reaction - restriction frag- ment length polymorphism (PCR-RFLP)}

In addition to PCR, for PCR-RFLP digestion with restriction enzyme is needed, and the obtained restriction fragments (FR) are separated based on their lengths by the aid of agarose gel electrophoresis. SNPs or Insertion/ Deletion (InDel) may abolish or lead to a new restriction endonuclease recognition sites, therefore it may affect the quantity and the length of DNA fragments resulting from restriction enzyme digestion. PCR-RFLP is frequently used in genotyping, for hereditary disease diagnostics, etc. For example, PCR-RFLP method was used for identification of point mutation (N370S, L444P, 84GG, R463C) in GBA gene that results in Gaucher disease (6). MDR1 (or ABCB1) gene polymorphisms C1236T, G2677T, and C3435T were genotyped by PCR-RFLP techniques to investigate the association between the mentioned SNPs and antiepileptic drug concentrations and treatment response (7), clinical course of pediatric pulmonary arterial hypertension (8), and gastric lesions (9). Furthermore, the PCR-RFLP method was applied for analyzing the interleukin-6 SNPs and their correlations with patients' sepsis risk and severity in adult patients (10) for glutathione S-transferase (GST) SNPs in premalignant gastric lesions risk (11) and for investigation of the impact of CYP2C19 polymorphisms in platelet response to clopidogrel (12) and on severity of gastric lesions (9).

Nested PCR technique is a variant of PCR that increases the specificity and efficiency of the desired amplicons by using two pairs of PCR primers (outer primers and nested primers).

Amplification-Refractory Mutation System Polymerase chain reaction (ARMS-PCR) analysis is a simple method used to identify single base pair mutations. Nested PCR and ARMSPCR were routinely used for investigation of c.35delG and pW24X mutations in GJB2 gene 179 children with non-syndromic hearing loss (NSHL) (13).

Multiplex-PCR technique is a fast method that allows simultaneous amplification of different DNA sequences (targets) in a single PCR tube. Multiplex-PCR may be used for SNP genotyping, mutation analysis, gene deletion analysis. For example it was applied for detection of del(GJB6-D13S1830) and del(GJB6-D13S1854) in GJB6 gene in 179 Romanian NSHL children (13).

Reverse transcriptase polymerase chain reaction (RT-PCR) is used to detect the level of RNA transcripts. RT-PCR may also be utilized for quantification of RNA by incorporating quantitative real-time PCR. Therefore, real-time RT-PCR is widely used in hematological malignancies. For example, real-time RT-PCR was used for identification of fusion gene transcripts (E2A-PBX1, MLL-AF4, TEL-AML1, BCR-ABL1, SIL-TAL1) for residual disease detection in children with acute lymphoblastic leukemia (ALL) (1).

\section{Multiplex ligation dependent probe amplification (MLPA)}

MLPA is a method that allows to identify ab- 
errant copy numbers in up to 60 specific DNA sequences by using one PCR primer pair and performing a PCR reaction. MLPA may be used for the detection of exon deletions/duplications in different human genes, detection of aneuploidies (such as trisomy), for detection of a known point mutation (14), DNA methylation analysis for methylation profiling. MLPA may be applied for characterization of chromosomal abnormalities in tumor samples (http://www.mlpa. com). For example, MLPA is useful for investigation of the presence of large genomic rearrangements (deletions, duplications) in breast cancer patients (15).

Recently, it has been showed that MLPA is a valuable and fast method for identification of origin of sSMC identified by cytogenetic analysis in patients with developmental delay and congenital anomalies (congenital hearts defects) (5).

\section{Sanger sequencing}

Sanger sequencing is considered the "gold-standard" of DNA sequencing techniques. It is a "first-generation" DNA sequencing and represents an invaluable method in any clinical genomics laboratory because it is widely used for validation of next-generation sequencing results. In fact, it is a targeted sequencing method that uses oligonucleotide primers to search specific DNA sequences and provides an extensive mutational profiling of many genes of interest. One limitation of Sanger sequencing is represented by the fact that it is unable to detect mosaic alleles below a threshold of about $20 \%$.

Sanger sequencing may be used for confirmation of point mutation identified through MLPA analysis (14). The application of target sequencing revealed in exon 4 of NIPAL4 gene a homozygote missense mutation, namely c.527C $>$ A that led to Alanine-to-Aspartic acid substitution [NM_001099287.1(NIPAL4):c.527C >A (p.Ala176Asp)] (16). The mutation was reported to be pathogenic and to be associated with autosomal recessive congenital ichthyosis 6, ARCI6, OMIM 612281.

Sanger sequencing is largely used for the identification of somatic or germline mutations in cancer. In this respect, a new, robust, fast and cost-effective protocol for sequencing mismatch repair (MMR) genes was recently developed by Negura et al. (17). Hereditary Nonpolyposis Colorectal Cancer (HNPCC), also known as Lynch syndrome, is mainly attributable to germline mutations in the MSH2, MSH6, and MLH1 genes. The one plate system proposed will allow to personalize molecular oncogenetic diagnosis in Lynch syndrome in Romania (17).

\section{Next-generation sequencing (NGS)}

NGS is a new, high-throughput technology that allows rapid sequencing of the base pairs in DNA or RNA samples. In fact, NGS is a massively parallel sequencing technology and therefore is faster and cheaper than first-generation DNA sequencing. NGS allows whole-genome sequencing (WGS), whole-exome sequencing (WES), and investigation of a set of genes or specific genomic regions of interest (gene panel). Therefore, NGS is a priceless tool as it allows sequencing of thousands of genes in a single test and concomitantly identification of substitutions, small indels, copy number variations (CNVs), single nucleotide variants (SNVs). NGS may be used for molecular analysis, aneuploidy detection, gene expression profiling, detection of epigenetic changes (such as transcriptome sequencing or methylome sequencing).

A multigene panel (that allowed the analysis of the following genes ATM, BARD1, BLM, BRCA1, BRCA2, BRIP1, CDH1, CHEK2, FAM175A, MEN1, MLH1, MRE11A, MSH2, MSH6, MUTYH, NBN, PALB2, PMS2, PTEN, RAD50, RAD51C, RAD51D, STK11, TP53, XRCC2) was used for investigation of 80 Romanian patients 
with breast cancer. More than $50 \%$ of the mutations identified by NGS technology in Romanian female patients was localized in the $B R C A 1$ and $B R C A 2$ genes, the rest being found in genes CHEK2, PALB2, ATM and TP 53, but no pathogenic variants were identified in PTEN, STK11, and $\mathrm{CDH} 1$ genes (15).

A new assay to identify recurrent mutations in one region of $D N M T 3 A$, one region of $I D H 1$, one region of FLT3, two regions of $I D H 2$, and 5 regions of $R U N X 1$ in AML using next-generation sequencing was previously developed by Coriu et al. (18). The assay proposed by Coriu et al. was reported to be more cost-efficient and with a higher sensitivity of mutation detection than Sanger sequencing, it was supposed to be useful for quantification of mutation minimal residual disease (MRD) and it also allowed risk stratification of AML cases with normal karyotype (18).

In summary, genetic testing may represent a useful tool for diagnosis, monitoring of disorder in certain situations, such as a clinical suggestive phenotype for a genetic disorder or a specific type of malignancy or minimal residual disease. Unfortunately, some of them (i.e. sequencing techniques) are rather costly to be used for routine clinical practice.

\section{Conflict of interest}

None to declare.

\section{References}

1. Jinca C, Petrescu CAM, Boeriu E, Oprisoni A, BalintGib L, Baica M, et al. The impact of immunological and biomolecular investigations on the outcome of children with acute lymphoblastic leukemia - experience of IIIrd Paediatric Clinic Timisoara. Rev Romana Med Lab. 2018;26(1):77-85. DOI: 10.1515/rrlm-2017-0029

2. Arber DA, Orazi A, Hasserjian R, Thiele J, Borowitz MJ, Le Beau MM, et al. The 2016 revision to the World Health Organization classification of myeloid neoplasms and acute leukemia. Blood.
2016 May 19;127(20):2391-405. DOI: 10.1182/ blood-2016-03-643544

3. Döhner H, Estey E, Grimwade D, Amadori S, Appelbaum FR, Büchner T, et al. Diagnosis and management of AML in adults: 2017 ELN recommendations from an international expert panel. Blood. 2017 Jan 26;129(4):424-47 DOI: 10.1182/ blood-2016-08-733196

4. Berbec NP, Papuc SM, Tutulan-Cunita AC, Angelescu SM, Lupu AI, Arghir AA. Molecular characterization of complex chromosomal changes in de novo acute myeloid leukemia: a case report. Rev Romana Med Lab. 2013;21(1):59-65. DOI: 10.2478/rrlm-2013-0018

5. Crauciuc GA, Tripon F, Bogliş A, Făgărăşan A, Bănescu C. Multiplex ligation dependent probe amplification - A useful, fast and cost-effective method for identification of small supernumerary marker chromosome in children with developmental delay and congenital heart defect. Rev Romana Med Lab. 2018;26(4):461-70. DOI: $10.2478 / \mathrm{rrlm}-2018-0032$

6. Drugan C, Drugan T, Caillaud C, Grigorescu-Sido P, Nistor T, Crăciun AM. Laboratory diagnosis and follow-up of Romanian Gaucher disease patients. Rev Romana Med Lab. 2017;25(3):275-85. DOI: 10.1515/ rrlm-2017-0018

7. Sabin O, Bocșan IC, Trifa A, Major ZZ, Heghes SC, Brusturean Bota E, et al. Correlation between ABCB1 gene polymorphisms, antiepileptic drug concentrations and treatment response. Rev Romana Med Lab. 2018;26(4):479-87. DOI: 10.2478/rrlm-2018-0012

8. Muntean I, Şuteu C, Togănel R, Bănescu C. Association between MDR1 gene polymorphism and clinical course of pediatric pulmonary arterial hypertension. Rev Romana Med Lab. 2018;26(3):305-12. DOI: 10.2478/ rrlm-2018-0025

9. Negovan A, Iancu M, Moldovan V, Pantea M, Sarkany K, Bataga S, et al. Influence of MDR1 C3435T, CYP2C19*2 and CYP2C19*3 gene polymorphisms and clinical characteristics on the severity of gastric lesions: a case-control study. J Gastrointestin Liver Dis. 2016;25(2):258-60. DOI: 10.15403/ jgld.2014.1121.252.mdr.

10. Georgescu AM, Bănescu C, Badea I, Moldovan V, Huțanu A, Voidăzan S, et al. IL-6 gene polymorphisms and sepsis in ICU adult Romanian patients: a prospective study. Rev Romana Med Lab. 2017;25(1):75-89. DOI: 10.1515/rrlm-2016-0044 
11. Negovan A, Iancu M, Moldovan V, Mocan S, Banescu C. The Interaction between GSTT1, GSTM1, and GSTP1 Ile105Val Gene Polymorphisms and Environmental Risk Factors in Premalignant Gastric Lesions Risk. Biomed Res Int. 2017;2017:7365080. DOI: 10.1155/2017/7365080.

12. Mărginean A, Bănescu C, Moldovan V, Scridon A, Mărginean M, Bălaşa R, et al. The Impact of CYP2C19 Loss-of-Function Polymorphisms, Clinical, and Demographic Variables on Platelet Response to Clopidogrel Evaluated Using Impedance Aggregometry. Clin Appl Thromb Hemost. 2017;23(3):255-65. DOI: 10.1177/1076029616629211.

13. Lazăr C, Popp R, Al-Khzouz C, Mihuț G, Grigorescu-Sido P. GJB2 and GJB6 genes mutations in children with non-syndromic hearing loss. Rev Romana Med Lab. 2017;25(1):37-46. DOI: 10.1515/rrlm-2017-0004

14. Bogliş A, Tripon F, Bănescu C. The utility of molecular genetic techniques in craniosynostosis cases associated with intellectual disability. Rev Romana Med Lab.
2018;26(4):471-7. DOI: 10.2478/rrlm-2018-0033

15. Goidescu IG, Eniu DT, Caracostea GV, Cruciat G, Stamatian F. Associations of pathogenic mutations responsible for breast cancer risk with histology and immunohistochemistry in Romanian population. Rev Romana Med Lab. 2018;26(2):165-75. DOI: 10.1515/ rrlm-2017-0037

16. Maier D, Florea A, Tilinca MC, Zazgyva A, Cosgarea R. NIPAL4 mutation c.527C $>$ A identified in Romanian patients with autosomal recessive congenital ichthyosis. Rev Romana Med Lab. 2016;24(4):387-97. DOI: 10.1515/rrlm-2016-0034

17. Negura L, Negura A. Sanger sequencing of MMR genes in a one-plate system. Rev Romana Med Lab. 2018;26(2):153-63. DOI: 10.2478/rrlm-2018-0008

18. Coriu D, Jardan D, Jardan C, Tălmaci R, Dragomir M, Coliţă A. A new assay to identify recurrent mutations in acute myeloid leukemia using next-generation sequencing. Rev Romana Med Lab. 2014;22(1):93-9. DOI: $10.2478 / \mathrm{rrlm}-2014-0003$ 